\title{
Modeling Subgrid Viscosity for Advection-Diffusion Problems
}

\author{
F. Brezzi \\ Dipartimento di Matematica and I.A.N.-C.N.R. \\ Via Abbiategrasso 215, 27100 Pavia (Italy) \\ P. Houston \\ Department of Mathematics \& Computer Science \\ University of Leicester, Leicester LE1 $7 R H$ (UK) \\ D. Marini \\ Dipartimento di Matematica and I.A.N.-C.N.R. \\ Via Abbiategrasso 215, 27100 Pavia (Italy) \\ E. Süli \\ Oxford University Computing Laboratory \\ Wolfson Building, Parks Road, Oxford OX1 3QD (UK)
}

\begin{abstract}
We analyse the effect of the subgrid viscosity on a finite element discretisation, with piecewise linear elements, of a linear advection-diffusion scalar equation. We point out the importance of a proper tune-up of the viscosity coefficient, and we propose a heuristic method for obtaining reasonable values for it. The extension to more general problems is then hinted in the last section.
\end{abstract}

E. Sïli and P. Houston acknowledge the financial support of the EPSRC (Grant GR/K76221).

Oxford University Computing Laboratory

Numerical Analysis Group

Wolfson Building

Parks Road

Oxford, England OX13QD

August, 1999 


\section{Introduction}

In a series of recent papers (see $[9,10,11]$, for example), P. Guermond proposed the use of subgrid viscosity methods in order to stabilise finite element discretisations of a variety of problems for which the standard Galerkin approach usually fails. The basic underlying idea is the following. We consider a finite element space $V_{h}$ that can be written as the direct sum of a coarse grid space $V_{C}$ and a finer (subgrid) space $V_{S}$. Assume that the standard Galerkin discretisation has the form

$$
a\left(u_{h}, v_{h}\right)=\left(f, v_{h}\right) \quad \forall v_{h} \in V_{h} .
$$

Then, roughly speaking, the subgrid viscosity method would consist of considering the stabilised form

$$
a\left(u_{h}, v_{h}\right)+C_{h} d\left(u_{S}, v_{S}\right)=\left(f, v_{h}\right) \quad \forall v_{h} \in V_{h},
$$

where, $u_{S}$ and $v_{S}$ are the subgrid components of $u_{h}$ and $v_{h}$, respectively, $d(u, v)$ is a "diffusion" term, and $C_{h}$ is a parameter to be chosen properly. This will, of course, be made more precise for a specific example in the next section. In the original presentation of Guermond, not much emphasis was given to the choice of the parameter $C_{h}$. The choice was usually of the type $C_{h}=c h$, and a priori error estimates were proven for a large range of possible values of the constant $c$. In our opinion, the idea is quite appealing, since it could be extended to a great variety of different important problems in which the implementation of other stabilising procedures, as for instance the Streamline-Upwind/Petrov-Galerkin (SUPG) method (see, for example, [14,12]), might become cumbersome, in particular compared with the striking simplicity of (1.2). On the other hand we believe that, disregarding for a while the asymptotic error analysis, for commonly used values of the coarse grid meshsize $h$ the choice of the subgrid viscosity parameter $C_{h}$ can become crucial, and that a bad choice for it can result in quite a poor method. In particular, as we are going to demonstrate in the present paper, the use of subgrid viscosity corresponds to using a residual-based stabilisation very similar to SUPG (and often coinciding with it), with an "intrinsic time scale" $\tau$ strongly dependent on the choice of $C_{h}$. Hence, the choice of the optimal value for $C_{h}$, or at least of a reasonably good one, appears to be of paramount importance. The problem of selecting an appropriate value for $C_{h}$ is not simple. Ideally, we would like to have an automatic procedure for choosing it, that is simple enough to be adapted to different physical applications and, if possible, be reasonably cheap. In this paper we propose a simple and cheap procedure that works reasonably well on the model problem of advection-dominated diffusion flows. For this problem, several heuristic rules have been proposed for the automatic choice of the parameter $\tau$ (see Brezzi \& Russo [3], Fischer et al. [4], Franca et al. [5,6], Führer \& Rannacher [8], Hansbo \& Johnson [12] Hughes \& Brooks [14] and Roos et al. [18], for example). The only derivation of a good value for $\tau$ which is based on the use of subgrid functions vanishing at the interelement boundary is, however, the one of [3] based on the residual free bubbles. The values of $\tau$ generated by our procedure are indeed in good agreement with the ones that could be obtained with the residual free bubble approach. We are fully aware that the linear advection-diffusion model problem cannot represent most of the difficulties that arise in real life problems. However, some generalisations of our idea to more complicated cases seem to be pretty 
obvious, while others will require further attention. But, of course, even the 'obvious' ones should be tested in practice before making claims. Despite these considerations, we still believe that it is important to point out the problems, together with a starting point for their possible solution.

For other attempts of an automatic tune-up of viscosity parameters we refer for instance to Brezzi et al. [1,2], Franca [5], Franca \& Russo [7], Hughes [13] and Oñate [16,17].

The outline of this paper is as follows. In Section 2 we first outline the subgrid viscosity method introduced by Guermond for an advection-diffusion model problem. In Section 3, we design a simple and cheap algorithm for automatically determining the size of the subgrid viscosity $C_{T}$. The practical performance of this procedure is then demonstrated in Section 4 for a simple advection-diffusion test problem. Finally, in Section 5 we summarise the work presented in this paper and draw some conclusions.

\section{Subgrid viscosity and its effects on the coarse grid}

In this section we are going to recall the basic idea of the subgrid viscosity method proposed by Guermond, and to show the importance of the choice of the subgrid viscosity parameter. In order to simplify the exposition, we consider a very simple model problem.

Suppose that $\Omega$ is a bounded polyhedral domain in $\mathbf{R}^{2}$ (but the extension to $\mathbf{R}^{n}$ would be straightforward), whose diameter has been scaled to 1 ; let $\beta$ be a constant vector field in $\Omega, \varepsilon$ a small parameter (compared with $|\beta|$ ) and $f$ a function in $L^{2}(\Omega)$ that we assume to be piecewise constant. We consider the problem of finding $u \in H_{0}^{1}(\Omega)$ such that

$$
-\varepsilon \Delta u+\beta \cdot \nabla u=f \quad \text { in } \Omega .
$$

Existence and uniqueness of the solution of (2.1) are well known. We assume now that we are given a triangulation of $\Omega$ into triangles $T$ of diameter $h_{T}$, and let $V_{C}$ be the space of continuous piecewise linear functions vanishing on $\partial \Omega$. On each triangle $T$ in the mesh, we define $V_{S}$ to be the space of continuous functions which vanish at the boundary of $T$. For simplicity, we assume that the space $V_{S}$ has only one degree of freedom for every triangle $T$ in the mesh. Particular examples include: given a triangle $T$, we can split $T$ into three subtriangles $T_{k}(k=1,2,3)$ by connecting the three vertices with the barycenter. Then, the space $V_{S} \equiv V_{S, L}$ may be constructed to consist of functions which are continuous and piecewise linear on the new triangulation and vanish at all the vertices of the old one, cf. Figure 1(a). Alternatively, we may define $V_{S} \equiv V_{S, C}$ to be the space of cubic bubbles on $T$ centered at the barycenter of the triangle, cf. Figure 1(b).

Before we proceed, it will be convenient to introduce some notation: for every subdomain $E \subseteq \Omega$, we write

$$
\begin{aligned}
& a_{E}(u, v)=\varepsilon \int_{E} \nabla u \cdot \nabla v \mathrm{~d} x+\int_{E}(\beta \cdot \nabla u) v \mathrm{~d} x, \\
& d_{E}(u, v)=\int_{E} \nabla u \cdot \nabla v \mathrm{~d} x,
\end{aligned}
$$




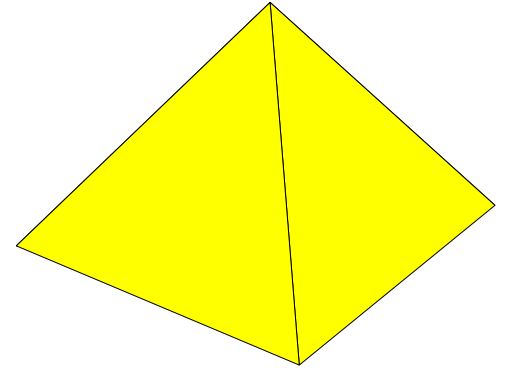

(a)

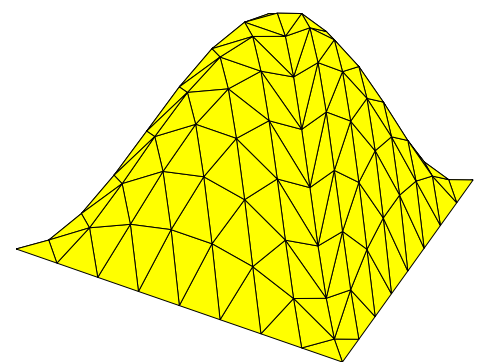

(b)

Figure 1: (a) Piecewise linear bubble; (b) Cubic bubble.

for $u, v$ in $H^{1}(E)$, and

$$
(u, v)_{E}=\int_{E} u v \mathrm{~d} x
$$

for $u, v$ in $L^{2}(E)$.

We consider now the space $V_{h}:=V_{C}+V_{S}$, and in $V_{h}$ we apply the following subgrid stabilisation: find $u_{h}=u_{C}+u_{S} \in V_{h}$ such that

$$
a_{\Omega}\left(u_{h}, v_{h}\right)+\sum_{T} C_{T} d_{T}\left(u_{S}, v_{S}\right)=\left(f, v_{h}\right)_{\Omega} \quad \forall v_{h}=v_{C}+v_{S} \in V_{h} .
$$

In [11] it is proved that optimal asymptotic error bounds for (2.2) hold, provided $C_{T}$ has the form $C_{T}=c_{T} h_{T}$, where $c_{T}$ is bounded from below and from above by fixed constants, independent of $T$ and $h_{T}$.

We want now to analyse the actual behaviour of (2.2) for different possible choices of $C_{T}$. For this we remark first that, for every $T$, we can define $p^{T}$ as the (unique) function in $V_{S}$ of height one, having support in $T$. Taking then $v_{h}=p^{T}$ in (2.2) we obtain:

$$
a_{T}\left(u_{C}+u_{S}, p^{T}\right)+C_{T} d_{T}\left(u_{S}, p^{T}\right)=\left(f, p^{T}\right)_{T} \quad \forall T .
$$

Setting now, in every $T, u_{S}=u^{T} p^{T}$ we then easily obtain from (2.3) that

$$
u^{T}\left(a_{T}\left(p^{T}, p^{T}\right)+C_{T} d_{T}\left(p^{T}, p^{T}\right)\right)=-a_{T}\left(u_{C}, p^{T}\right)+\left(f, p^{T}\right)_{T} \quad \forall T .
$$

We remark now that, for every $T$, the advective term has no effect in $a_{T}\left(p^{T}, p^{T}\right)$, being skew-symmetric in $H_{0}^{1}(T)$. Integrating by parts the first term in the right-hand side of (2.4) gives

$$
u^{T}=-\frac{\int_{T} R_{T} p^{T} \mathrm{~d} x}{\left(\varepsilon+C_{T}\right) \int_{T}\left|\nabla p^{T}\right|^{2} \mathrm{~d} x}=:-R_{T} \gamma_{T},
$$


where $R_{T}:=\left.\left(-\varepsilon \Delta u_{C}+\beta \cdot \nabla u_{C}-f\right)\right|_{T}$ is the residual (for the coarse grid solution) on the triangle $T$ and $\gamma_{T}$, as defined in (2.5), depends only on the bubble $p^{T}$ (plus $\varepsilon$ and $C_{T}$ ). We go back to $(2.2)$ where we take now $v_{h}=v_{C}$ :

$$
a_{\Omega}\left(u_{C}, v_{C}\right)+a_{\Omega}\left(u_{S}, v_{C}\right)=\left(f, v_{C}\right)_{\Omega} \quad \forall v_{C} \in V_{C},
$$

and we notice that (2.6) differs from the standard Galerkin finite element method on the coarse grid due to the presence of the stabilising term $a_{\Omega}\left(u_{S}, v_{C}\right)$. Let us analyse it in detail. Using (2.5) and integration by parts in $T$ we have

$$
a_{\Omega}\left(u_{S}, v_{C}\right)=\sum_{T} a_{T}\left(u^{T} p^{T}, v_{C}\right)=-\sum_{T} R_{T} \gamma_{T} L_{T}^{*} v_{C}\left(p^{T}, 1\right)_{T},
$$

where $L_{T}{ }^{*} v_{C}$ is defined as $-\varepsilon \Delta v_{C}-\beta \cdot \nabla v_{C}$ in every $T$. We obtain therefore

$$
a_{\Omega}\left(u_{S}, v_{C}\right)=-\sum_{T} \tau_{T}\left(R_{T}, L_{T}^{*} v_{C}\right)_{T},
$$

with the intrinsic time scale $\tau_{T}$ defined as

$$
\tau_{T}=\frac{1}{|T|} \frac{\left(\int_{T} p^{T} \mathrm{~d} x\right)^{2}}{\left(\varepsilon+C_{T}\right) \int_{T}\left|\nabla p^{T}\right|^{2} \mathrm{~d} x} .
$$

A simple computation shows that when $V_{S}=V_{S, L}$, the value of $\tau_{T}$ given by (2.8) is actually

$$
\tau_{T} \equiv \tau_{T, L}=\frac{4|T|^{2}}{27\left(\varepsilon+C_{T}\right) \sum_{j} e_{j}^{2}},
$$

where the $e_{j}(j=1,2,3)$ are the lengths of the edges of $T$. Alternatively, when $V_{S}=V_{S, C}$, we have

$$
\tau_{T} \equiv \tau_{T, C}=\frac{|T|^{2}}{10\left(\varepsilon+C_{T}\right) \sigma}
$$

where

$$
\sigma=\left(\mathrm{x}_{3}-\mathrm{x}_{1}\right) \cdot\left(\mathrm{x}_{1}-\mathrm{x}_{2}\right)+\left|\mathrm{x}_{3}-\mathrm{x}_{1}\right|^{2}+\left|\mathrm{x}_{2}-\mathrm{x}_{1}\right|^{2},
$$

and $\mathbf{x}_{i}=\left(x_{i}, y_{i}\right), i=1, \ldots, 3$, denote the coordinates of the three vertices of element $T$. To fix the ideas, on an equilateral triangle we would have

$$
\tau_{T, L}=\frac{h_{T}^{2}}{108\left(\varepsilon+C_{T}\right)}, \quad \tau_{T, C}=\frac{3 h_{T}^{2}}{400\left(\varepsilon+C_{T}\right)},
$$

respectively. The effect of the subgrid viscosity becomes now clear, as well as the role of the parameter $C_{T}$. Indeed, first of all, we have from (2.6) and (2.7) that the coarse equation (after condensation of the subgrid unknowns $u_{S}$ ) becomes

$$
a_{\Omega}\left(u_{C}, v_{C}\right)-\sum_{T} \tau_{T}\left(R_{T}, L_{T}{ }^{*} v_{C}\right)_{T}=\left(f, v_{C}\right)_{\Omega} \quad \forall v_{C} \in V_{C},
$$


and hence coincides with the SUPG method with a particular choice of the parameter $\tau_{T}$ given by (2.8) and depending on the choice of $C_{T}$. On the other hand, assuming that the natural viscosity $\varepsilon$ is very small, and that $C_{T}$ is chosen (as indicated in [11]) of the form $C_{T}=c_{T} h_{T}$, we would have, in (2.9), respectively, (2.10),

$$
\tau_{T, L} \approx \frac{1}{c_{T}} \frac{4|T|^{2}}{27 h_{T} \sum_{j} e_{j}^{2}}, \quad \tau_{T, C} \approx \frac{1}{c_{T}} \frac{|T|^{2}}{10 h_{T} \sigma},
$$

or, in (2.11),

$$
\tau_{T, L} \approx \frac{1}{c_{T}} \frac{h_{T}}{108}, \quad \tau_{T, C} \approx \frac{1}{c_{T}} \frac{3 h_{T}}{400},
$$

for equilateral triangles, respectively. Equations (2.12) and (2.13) show clearly the following fact, that would have been somehow counter-intuitive in the first place: the bigger one takes $c_{T}$ in the artificial subgrid viscosity, the smaller becomes the stabilising effect on the coarse grid equation.

In our opinion, this indicates also, in a very clear manner, that the choice of a good value for $c_{T}$ is extremely important for having satisfactory results on affordable grids. Actually, it is known that the tune-up of the $\tau_{T}$ in SUPG is quite important for that, and (2.12) shows that this problem is not solved by the subgrid viscosity approach.

\section{Tune-up of the subgrid viscosity}

We have seen in the previous section that the subgrid viscosity method suffers a major drawback. The precise amount of subgrid viscosity to be used is not at all clear, but, in spite of asymptotic results, the choice is actually crucial for obtaining reasonable performances on affordable grids. On the other hand, as we pointed out in the introduction, the method still remains appealing for its great simplicity, and in particular for the possibility to apply it, possibly without proofs, on complicated problems. For a very promising attempt in this direction, related to LES and turbulence, see Hughes et al. [15].

In this section we make an attempt to design a viable procedure that is able to produce, if not the optimal value to be used for $c_{T}$, at least a reasonable value. For the sake of simplicity, we present it on the simple model problem (2.1). However, the underlying idea is that, contrary to what was done previously for the choice of $\tau_{T}$ for the same model problem (see, for example, [1] and the references therein), here we make an effort to not use any a priori information on the nature of the underlying partial differential equation. More realistically, let us say that we are trying to minimise the use of a priori information on the problem.

The basic idea which is behind our strategy is the following. The subgrid component of the solution, $u_{S}$, can be thought of as being obtained by multiplying, element by element, the scalar constant factor $R_{T}$ (the residual of the coarse grid equation in element $T$ ) times the solution $w_{S}$ of the scaled subgrid equation

$$
a_{T}\left(w_{S}, v_{S}\right)+c_{T} h_{T} d_{T}\left(w_{S}, v_{S}\right)=\left(1, v_{S}\right)
$$


If the tune-up of $c_{T}$ is reasonably good, the solution should be a reasonable approximation of the solution of the corresponding continuous problem

$$
a_{T}\left(w_{S}^{e x}, v_{S}\right)=\left(1, v_{S}\right) \forall v_{S} \in H_{0}^{1}(T),
$$

where $w_{S}^{e x}$ is looked for in $H_{0}^{1}(T)$ as well. Being, as in our previous case, a one-degreeof-freedom approximation of (3.2), we cannot expect (3.1) to be too precise. We would however at least be satisfied if it produced a reasonably good approximation of the average of the solution over the triangle $T$. This seems particularly relevant, as it can be seen in our model case that the average of the solution plays a crucial role. Indeed, the solution $w_{S}$ of (3.1) satisfies, in our model problem,

$$
w_{S}=\frac{\int_{T} p^{T} \mathrm{~d} x}{\left(\varepsilon+C_{T}\right) \int_{T}\left|\nabla p^{T}\right|^{2} \mathrm{~d} x} p^{T},
$$

and comparing (3.3) with (2.8) we immediately obtain

$$
\tau_{T}=\frac{1}{|T|} \int_{T} w_{S} \mathrm{~d} x
$$

while, on the other hand, the residual-free bubble approach applied to the model problem would actually indicate as optimal $\tau_{T}$ the average of $w_{S}^{e x}$. Moreover, always if the tune-up of the subgrid viscosity parameter $c_{T}$ is reasonably good, any sub-subgrid approximation of the continuous equation (3.2), obtained making use of an artificial viscosity based on $c_{T}$, should also produce a solution $w_{S S}$ such that

$$
\frac{1}{|T|} \int_{T} w_{S S} \mathrm{~d} x \approx \frac{1}{|T|} \int_{T} w_{S} \mathrm{~d} x \approx \frac{1}{|T|} \int_{T} w_{S}^{e x} \mathrm{~d} x
$$

Equation (3.4) is the key point in our strategy. More precisely, the idea is to compute the solutions $w_{S}$ and $w_{S S}$ (on the subgrid and on the sub-subgrid, respectively) as functions of $c_{T}$, and then compute the desired value of $c_{T}$ by requiring that

$$
\frac{1}{|T|} \int_{T} w_{S S}\left(c_{T}\right) \mathrm{d} x \approx \frac{1}{|T|} \int_{T} w_{S}\left(c_{T}\right) \mathrm{d} x .
$$

It is clear that, taking the sub-subgrid mesh very fine, the function

$$
c_{T} \rightarrow \frac{1}{|T|} \int_{T} w_{S S}\left(c_{T}\right) \mathrm{d} x
$$

will become practically flat: on a very fine grid, every choice of $c_{T}$ would indeed give $w_{S S}$ practically equal to the exact solution. As the function

$$
c_{T} \rightarrow \frac{1}{|T|} \int_{T} w_{S}\left(c_{T}\right) \mathrm{d} x
$$

is monotonically decreasing, the equation

$$
\frac{1}{|T|} \int_{T} w_{S S}\left(c_{T}\right) \mathrm{d} x=\frac{1}{|T|} \int_{T} w_{S}\left(c_{T}\right) \mathrm{d} x
$$


will have a unique solution. Moreover, always in the ideal case in which the sub-subgrid is very fine, the solution of $(3.5)$ will provide a $c_{T}$ that, when used, will give a $w_{S}$ (and hence a $u_{S}$ ) with the "ideal" value for the integral. In practice, however, this would be tremendously expensive, and we shall use (3.5) with a sub-subgrid that is just slightly finer than the original one.

We end this section by outlining the practical implementation of the proposed algorithm for determining the subgrid viscosity $C_{T}$ on each element $T$ in the mesh. As previously stated we assume that $C_{T}=c_{T} h_{T}$, where $h_{T}$ is a measure of the length scale of element $T$. To this end, we first recall that for a general bubble function $p^{T}$, we have that

$$
\frac{1}{|T|} \int_{T} w_{S} \mathrm{~d} x=\frac{1}{|T|} \frac{\left(\int_{T} p^{T} \mathrm{~d} x\right)^{2}}{\left(\varepsilon+c_{T} h_{T}\right) \int_{T}\left|\nabla p^{T}\right|^{2} \mathrm{~d} x} .
$$

Thereby, by exploiting equation (3.5), we get

$$
c_{T} h_{T}=\frac{\left(\int_{T} p^{T} \mathrm{~d} x\right)^{2}}{\int_{T}\left|\nabla p^{T}\right|^{2} \mathrm{~d} x \int_{T} w_{S S}\left(c_{T}\right) \mathrm{d} x}-\varepsilon .
$$

Equation (3.7) may be used to determine the subgrid viscosity coefficient $c_{T}$ using the following iteration: select an initial guess $c_{T, 0}$, then for $j=0, \ldots, \widehat{n}$, set

$$
c_{T, j+1}=\frac{1}{h_{T}}\left(\frac{\left(\int_{T} p^{T} \mathrm{~d} x\right)^{2}}{\int_{T}\left|\nabla p^{T}\right|^{2} \int_{T} w_{S S}\left(c_{T, j}\right) \mathrm{d} x}-\varepsilon\right) .
$$

Here, $\widehat{n}$ is the smallest number of iterations required to ensure that the convergence criterion:

$$
\left|c_{T, \widehat{n}}-c_{T, \widehat{n}-1}\right| \leq \mathrm{TOL}
$$

is satisfied. For the practical implementation of this algorithm we choose TOL to be equal to $\varepsilon / 10 h_{T}$; i.e. the subgrid viscosity $C_{T}\left(=c_{T} h_{T}\right)$ is calculated to within an accuracy of an order of magnitude less than the underlying physical diffusion. We remark that this tolerance level is sufficient to compute $c_{T}$ accurately with a small amount of computational effort.

\section{Numerical Example}

In this section we present a numerical experiment in 2 space dimensions to compare the performance of the subgrid stabilisation algorithm outlined in the previous section, using both the piecewise linear bubble function and the cubic bubble, with the residual free bubble approach. We recall, that the latter approach gives rise to the following SUPG stabilisation parameter

$$
\tau_{T} \equiv \tau_{T, R}=\frac{1}{|T|} \int_{T} w_{S}^{e x} \mathrm{~d} x
$$




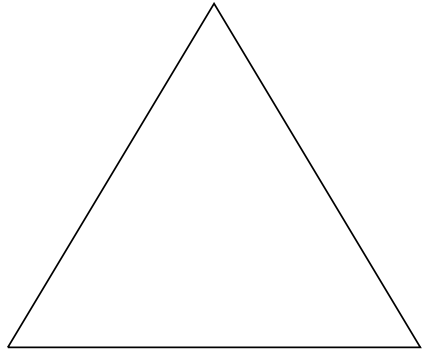

(a)

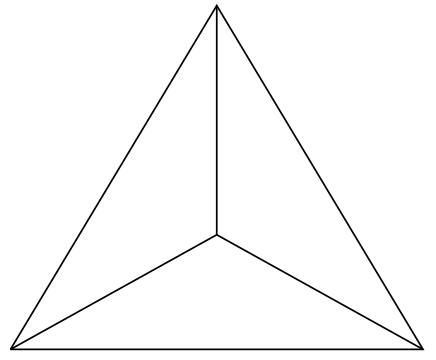

(b)

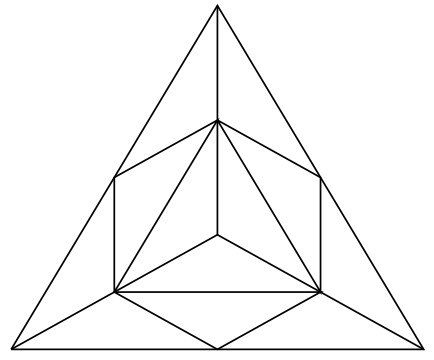

(c)

Figure 2: (a) Element T; (b) Subgrid; (c) Sub-subgrid.

where $w_{S}^{e x}$ is the analytical solution of the subgrid equation (3.2) on each element $T$ in the mesh. To this end, we consider the numerical example presented by Fischer et al. [4]; here, $\Omega=(0,1)^{2}, \beta$ is chosen so the flow is at angle of $22^{\circ}$ to the horizontal (i.e. $\beta=(-0.927,0.375))$ and $f=0$ with boundary condition

$$
u(x, y)= \begin{cases}0 & \text { for } x=0,0 \leq y \leq 1 \\ 0 & \text { for } 0 \leq x \leq 1, y=1 \\ 0 & \text { for } 0 \leq x<1 / 2, y=0 \\ 1 & \text { for } 1 / 2 \leq x \leq 1, y=0 \\ 1 & \text { for } x=1,0 \leq y \leq 1\end{cases}
$$

We begin by first comparing the performance of the subgrid stabilisation method using the piecewise linear bubble with the residual free bubble approach. Given a triangle $T$ in the mesh, cf. Figure 2(a), we recall that the subgrid solution $w_{S}$ is computed on a mesh with one interior node located at the centroid of $T$, cf. Figure 2(b). For computational efficiency, the sub-subgrid solution $w_{S S}$ will be computed on a mesh based on one uniform refinement of the subgrid mesh; thereby, $w_{S S}$ is calculated on a mesh with four interior nodes, $\mathrm{cf}$. Figure 2(c). In Tables $1,2 \& 3$, we present numerical results for $\varepsilon=10^{-1}, 2 \times 10^{-3} \&$ $10^{-5}$, respectively. In each case, we compute the mesh Peclét number $\operatorname{Pe}(=|\beta| h / \varepsilon)$, the subgrid viscosity $C_{T}$, the stabilisation parameter using the piecewise linear bubble, $\tau_{T, L}$, the stabilisation parameter arising from the residual free bubble approach, $\tau_{T, R}$, and the ratio $\tau_{T, L} / \tau_{T, R}$, on a sequence of uniform triangular meshes. We note that, in each case the mesh is constructed from a uniform $N \times N$ mesh by connecting the bottom-left corner of each mesh square with its top-right corner. Furthermore, the stabilisation parameter $\tau_{T, R}$ is calculated by numerically approximating the subgrid equation (3.2) using the standard Galerkin finite element method on a fine mesh partition of each triangle $T$.

From Table 1, we observe that when the mesh Peclét number is small $(\mathrm{Pe}<2)$, the subgrid viscosity $C_{T}$ is $\mathcal{O}(1)$ as the mesh is refined. In fact, here $C_{T}$ is actually negative, though by construction, the total effective viscosity, namely $\varepsilon+C_{T}$, is guaranteed to be strictly positive, cf. (3.7). Furthermore, the stabilisation parameters arising from the piecewise linear bubble and the residual free bubble, $\tau_{T, L}$ and $\tau_{T, R}$, respectively, are both $\mathcal{O}\left(h^{2}\right)$ as $h$ tends to 0 , with $\tau_{T, R}$ being slightly larger than $\tau_{T, L}$ by a consistent factor. In 


\begin{tabular}{||c|c|c|c|c|c||}
\hline Mesh & Pe & $C_{T}$ & $\tau_{T, L}$ & $\tau_{T, R}$ & $\tau_{T, L} / \tau_{T, R}$ \\
\hline $17 \times 17$ & 0.63 & $-1.640 \times 10^{-2}$ & $4.342 \times 10^{-4}$ & $5.090 \times 10^{-4}$ & 0.85 \\
\hline $33 \times 33$ & 0.31 & $-1.674 \times 10^{-2}$ & $1.086 \times 10^{-4}$ & $1.273 \times 10^{-4}$ & 0.85 \\
\hline $65 \times 65$ & 0.16 & $-1.675 \times 10^{-2}$ & $2.715 \times 10^{-5}$ & $3.184 \times 10^{-5}$ & 0.85 \\
\hline $129 \times 129$ & 0.08 & $-1.665 \times 10^{-2}$ & $6.780 \times 10^{-6}$ & $7.961 \times 10^{-6}$ & 0.85 \\
\hline $257 \times 257$ & 0.04 & $-1.639 \times 10^{-2}$ & $1.690 \times 10^{-6}$ & $1.990 \times 10^{-6}$ & 0.85 \\
\hline
\end{tabular}

Table 1: Stabilisation parameters $\tau_{T, L}$ and $\tau_{T, R}$ for $\varepsilon=10^{-1}$.

\begin{tabular}{||c|c|c|c|c|c||}
\hline Mesh & Pe & $C_{T}$ & $\tau_{T, L}$ & $\tau_{T, R}$ & $\tau_{T, L} / \tau_{T, R}$ \\
\hline $17 \times 17$ & 31.3 & $1.348 \times 10^{-3}$ & $1.080 \times 10^{-2}$ & $1.165 \times 10^{-2}$ & 0.93 \\
\hline $33 \times 33$ & 15.6 & $2.054 \times 10^{-4}$ & $4.100 \times 10^{-3}$ & $4.423 \times 10^{-3}$ & 0.93 \\
\hline $65 \times 65$ & 7.81 & $-1.861 \times 10^{-4}$ & $1.246 \times 10^{-3}$ & $1.403 \times 10^{-3}$ & 0.89 \\
\hline $129 \times 129$ & 3.91 & $-2.968 \times 10^{-4}$ & $3.318 \times 10^{-4}$ & $3.842 \times 10^{-4}$ & 0.86 \\
\hline $257 \times 257$ & 1.95 & $-3.233 \times 10^{-4}$ & $8.426 \times 10^{-5}$ & $9.861 \times 10^{-5}$ & 0.85 \\
\hline
\end{tabular}

Table 2: Stabilisation parameters $\tau_{T, L}$ and $\tau_{T, R}$ for $\varepsilon=2 \times 10^{-3}$.

contrast, when the mesh Peclét number is very large, Table 3 indicates that $C_{T}, \tau_{T, L}$ and $\tau_{T, R}$ are all $\mathcal{O}(h)$ as the mesh is refined; here, $\tau_{T, L}$ is now roughly of the same size as $\tau_{T, R}$. In Table 2, we clearly see the transition between these two regimes as the mesh Peclét number decreases. This asymptotic behaviour of the stabilisation parameters $\tau_{T, L}$ and $\tau_{T, R}$ as the mesh is refined is consistent with the usual choice of $\tau_{T}$ for the SUPG scheme, predicted by standard a priori error analysis, cf. [18], for example.

Let us now investigate the behaviour of the subgrid stabilisation method using the cubic bubble. For computational simplicity, here the sub-subgrid solution $w_{S S}$ is again approximated on a mesh consisting of four interior nodes with piecewise linear basis functions, cf. Figure 2(c). In Tables 4, $5 \& 6$, we present a comparison of $\tau_{T, C}$ and $\tau_{T, R}$ for $\varepsilon=10^{-1}$, $2 \times 10^{-3} \& 10^{-5}$, respectively. Here, we observe that $\tau_{T, C}$ is consistently smaller than the stabilisation parameter predicted by the residual free bubble approach. Furthermore, by

\begin{tabular}{||c|c|c|c|c|c||}
\hline Mesh & $\mathrm{Pe}$ & $C_{T}$ & $\tau_{T, L}$ & $\tau_{T, R}$ & $\tau_{T, L} / \tau_{T, R}$ \\
\hline $17 \times 17$ & 6250 & $2.151 \times 10^{-3}$ & $1.674 \times 10^{-2}$ & $1.600 \times 10^{-2}$ & 1.05 \\
\hline $33 \times 33$ & 3125 & $1.086 \times 10^{-3}$ & $8.251 \times 10^{-3}$ & $7.972 \times 10^{-3}$ & 1.03 \\
\hline $65 \times 65$ & 1562.5 & $5.520 \times 10^{-4}$ & $4.022 \times 10^{-3}$ & $3.972 \times 10^{-3}$ & 1.01 \\
\hline $129 \times 129$ & 781.3 & $2.823 \times 10^{-4}$ & $1.933 \times 10^{-3}$ & $1.972 \times 10^{-3}$ & 0.98 \\
\hline $257 \times 257$ & 390.6 & $1.449 \times 10^{-4}$ & $9.122 \times 10^{-4}$ & $9.721 \times 10^{-4}$ & 0.94 \\
\hline
\end{tabular}

Table 3: Stabilisation parameters $\tau_{T, L}$ and $\tau_{T, R}$ for $\varepsilon=10^{-5}$. 


\begin{tabular}{||c|c|c|c|c|c||}
\hline Mesh & $\mathrm{Pe}$ & $C_{T}$ & $\tau_{T, C}$ & $\tau_{T, R}$ & $\tau_{T, C} / \tau_{T, R}$ \\
\hline $17 \times 17$ & 0.63 & $2.742 \times 10^{-2}$ & $3.832 \times 10^{-4}$ & $5.090 \times 10^{-4}$ & 0.75 \\
\hline $33 \times 33$ & 0.31 & $2.737 \times 10^{-2}$ & $9.584 \times 10^{-5}$ & $1.273 \times 10^{-4}$ & 0.75 \\
\hline $65 \times 65$ & 0.16 & $2.736 \times 10^{-2}$ & $2.396 \times 10^{-5}$ & $3.184 \times 10^{-5}$ & 0.75 \\
\hline $129 \times 129$ & 0.08 & $2.752 \times 10^{-2}$ & $5.983 \times 10^{-6}$ & $7.961 \times 10^{-6}$ & 0.75 \\
\hline $257 \times 257$ & 0.04 & $2.796 \times 10^{-2}$ & $1.491 \times 10^{-6}$ & $1.990 \times 10^{-6}$ & 0.75 \\
\hline
\end{tabular}

Table 4: Stabilisation parameters $\tau_{T, C}$ and $\tau_{T, R}$ for $\varepsilon=10^{-1}$.

\begin{tabular}{||c|c|c|c|c|c||}
\hline Mesh & $\mathrm{Pe}$ & $C_{T}$ & $\tau_{T, C}$ & $\tau_{T, R}$ & $\tau_{T, C} / \tau_{T, R}$ \\
\hline $17 \times 17$ & 31.3 & $2.826 \times 10^{-3}$ & $1.012 \times 10^{-2}$ & $1.165 \times 10^{-2}$ & 0.87 \\
\hline $33 \times 33$ & 15.6 & $1.276 \times 10^{-3}$ & $3.726 \times 10^{-3}$ & $4.423 \times 10^{-3}$ & 0.84 \\
\hline $65 \times 65$ & 7.81 & $7.477 \times 10^{-4}$ & $1.111 \times 10^{-3}$ & $1.403 \times 10^{-3}$ & 0.79 \\
\hline $129 \times 129$ & 3.91 & $5.989 \times 10^{-4}$ & $2.936 \times 10^{-4}$ & $3.842 \times 10^{-4}$ & 0.76 \\
\hline $257 \times 257$ & 1.95 & $5.678 \times 10^{-4}$ & $7.428 \times 10^{-5}$ & $9.861 \times 10^{-5}$ & 0.75 \\
\hline
\end{tabular}

Table 5: Stabilisation parameters $\tau_{T, C}$ and $\tau_{T, R}$ for $\varepsilon=2 \times 10^{-3}$.

comparing $\tau_{T, C}$ with the size of $\tau_{T, L}$, we see that the stabilisation parameter using the cubic bubble is also smaller than the corresponding value using the piecewise linear bubble.

Finally, in Figure 3 we present the numerical solution to (2.1) for $\varepsilon=10^{-2}$, using each of the stabilisation methods discussed in this paper. In each case, the solution is computed on the unstructured triangular mesh shown in Figure 3(a). Here, we observe that each of the stabilisation schemes perform very well; over-shoots are observable in the boundary layers, but these may only be eradicated when the mesh Peclét number is large by the introduction of an isotropic artificial-diffusion model in addition to the streamline-diffusion stabilisation considered here, cf. [12].

\begin{tabular}{||c|c|c|c|c|c||}
\hline Mesh & $\mathrm{Pe}$ & $C_{T}$ & $\tau_{T, C}$ & $\tau_{T, R}$ & $\tau_{T, C} / \tau_{T, R}$ \\
\hline $17 \times 17$ & 6250 & $3.635 \times 10^{-3}$ & $1.340 \times 10^{-2}$ & $1.600 \times 10^{-2}$ & 0.84 \\
\hline $33 \times 33$ & 3125 & $1.818 \times 10^{-3}$ & $6.679 \times 10^{-3}$ & $7.972 \times 10^{-3}$ & 0.84 \\
\hline $65 \times 65$ & 1562.5 & $9.087 \times 10^{-4}$ & $3.322 \times 10^{-3}$ & $3.972 \times 10^{-3}$ & 0.84 \\
\hline $129 \times 129$ & 781.3 & $4.543 \times 10^{-4}$ & $1.643 \times 10^{-3}$ & $1.972 \times 10^{-3}$ & 0.83 \\
\hline $257 \times 257$ & 390.6 & $2.267 \times 10^{-4}$ & $8.060 \times 10^{-4}$ & $9.721 \times 10^{-4}$ & 0.83 \\
\hline
\end{tabular}

Table 6: Stabilisation parameters $\tau_{T, C}$ and $\tau_{T, R}$ for $\varepsilon=10^{-5}$. 


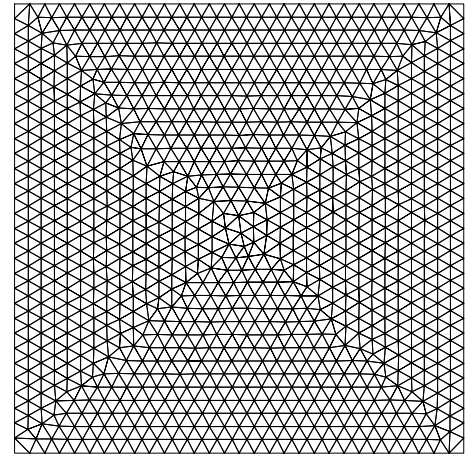

(a)

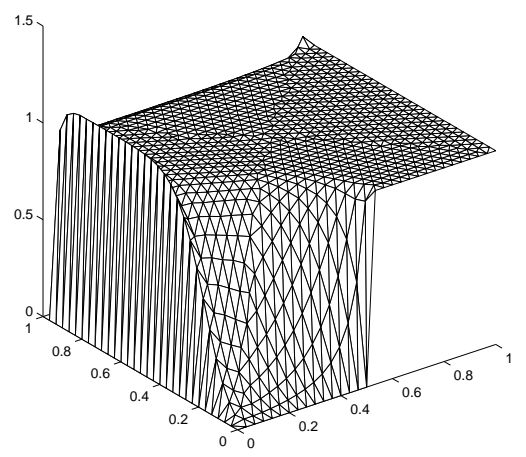

(c)

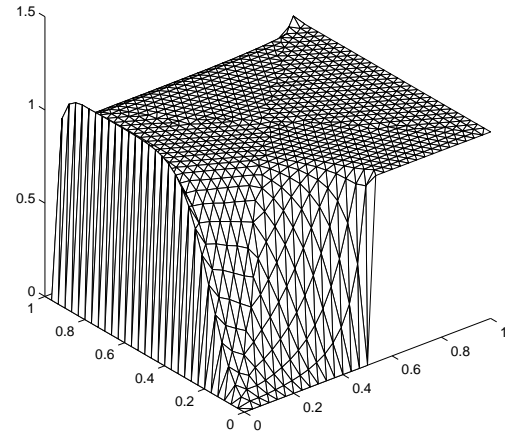

(b)

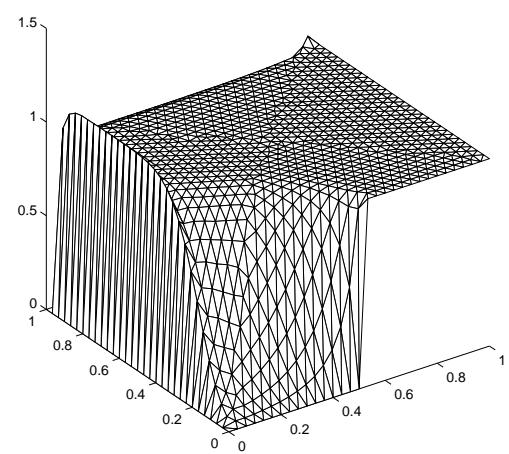

(d)

Figure 3: (a) Unstructured triangular mesh with 2046 elements and 1084 nodes. Numerical approximation using: (b) Residual free bubble stabilisation; (c) Piecewise linear bubble subgrid stabilisation; (d) Cubic bubble subgrid stabilisation.

\section{Concluding remarks}

In this paper we have analysed the effect of the subgrid viscosity method proposed by P. Guermond for stabilising the standard Galerkin finite element method for advectiondominated diffusion problems. In particular, we have shown that by increasing the amount of subgrid viscosity added into the scheme actually leads to a decrease in the overall stabilising effect on the coarse grid solution. Thereby, determining the amount of subgrid viscosity is extremely important for computing a good numerical approximation on practical meshes. Here, we have designed and implemented a simple and cheap algorithm for automatically determining the amount of subgrid viscosity. Moreover, our algorithm gives rise to a stabilisation parameter which is in good agreement with the one obtained from the residual free bubble approach. Extensions to more general problems, and in particular, to nonlinear problems are possible. However, this subject is beyond the scope of the current paper and will be presented elsewhere. 


\section{References}

[1] F. Brezzi, L. Franca, T..J.R. Hughes and A. Russo, $b=\int g$. Comput. Methods Appl. Mech. Engrg. 145:329-339, 1997.

[2] F. Brezzi, L.D. Marini And A. Russo, Application of pseudo residual-free bubbles to the stabilization of convection-diffusion problems. Comput. Methods Appl. Mech. Engrg. 166:51-63, 1998.

[3] F. Brezzi And A. Russo, Choosing bubbles for advection-diffusion problems. Math. Mod. and Meth. in Appl. Sci. 4:571-587, 1994.

[4] B. Fischer, A. Ramage, D..J. Silvester and A.J. Wathen, On parameter choice and iterative convergence for stabilised discretisations of advection-diffusion problems. Comput. Methods Appl. Mech. Engrg. (to appear).

[5] L.P. Franca (Editor). Special issue on Advances in Stabilized Methods in Computational Mechanics. Comput. Methods Appl. Mech. Engrg. 166, 1998.

[6] L.P. Franca, S.L. Frey And T..J.R. Hughes, Stabilised finite element methods: I. Application to the advection-diffusion model. Comput. Methods Appl. Mech. Engrg. 95:253-276, 1992.

[7] L.P. FRAnCA AND A. Russo, Deriving upwinding, mass lumping and selective reduced integration by residual-free bubbles. Appl. Math. Letters 9:83-88, 1996.

[8] C. FÜhrer and R. Rannacher, An adaptive streamline-diffusion finite element method for hyperbolic conservation laws. East-West J. Numer. Math. 5:145-162, 1997.

[9] J.-L. Guermond, Stabilization of Galerkin approximations of transport equations by subgrid modeling. Submitted to Math. Mod. Num. Anal., 1998.

[10] J.-L. Guermond, Stabilisation par viscosité de sous-maille pour l'approximation de Galerkin des opérateurs monotones. C.R. Acad. Sci. Paris, Séroe I, 328:617-622, 1999.

[11] J.-L. Guermond, Subgrid stabilization of Galerkin approximations of linear monotone operators. IMA J. Numer. Anal. (to appear).

[12] P. Hansbo And C. Johnson, Streamline diffusion finite element methods for fluid flow. von Karman Institute Lectures, 1995.

[13] T.J.R. Hughes, Multiscale phenomena: Green's functions, the Dirichlet-to-Neumann formulation, subgrid scale models, bubbles and the origin of stabilized methods. Comput. Methods Appl. Mech. Engrg. 127:387-401, 1995.

[14] T.J.R. Hughes And A. Brooks, A theoretical framework for Petrov-Galerkin methods with discontinuous weighting functions: application to the streamline-upwind procedure. In R. H. Gallagher, D.H. Norrie, J.T. Oden, and O.C. Zienkiewicz, editors, Finite Elements in Fluids, Volume 4, pages 47-65. John Wiley \& Sons, 1982. 
[15] T.J.R. Hughes, L. Mazzei And K.E. JAnsen, Large Eddy simulation and the variational multiscale method (submitted to Computing and Visualization in Science).

[16] E. OÑATE, Derivation of stabilized equations for advective-diffusive transport and fluid flow problems. Comput. Methods Appl. Mech. Eng., 151:233-267, 1998.

[17] E. OÑATE, J. García And S. Idelsohn, Computation of the stabilization parameter for the finite element solution of advective-diffusive problems. Int. J. Numer. Methods Fluids, 25:1385-1407, 1997.

[18] H.-G. Roos, M. Stynes And L. ToBiska, Numerical Methods for Singularly Perturbed Differential Equations. Convection-Diffusion and Flow Problems. Springer Series in Computational Mathematics, Volume 24, Springer-Verlag, 1996. 